Military Technical College

Kobry El-Kobbah,

Cairo, Egypt

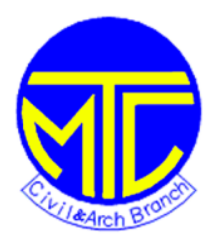

\title{
Buckling of slender prismatic columns with multiple edge cracks using energy approach
}

$$
\text { By }
$$

Talaat H. Abdel-lateef*

Magdy Israel Salama**

\section{Abstract:}

In this paper, the elastic stability of slender columns weakened by multiple edge cracks subjected to concentrated loads applied at the ends is presented. The analysis is carried out by using the minimum potential energy technique. The cracked section is modeled by a massless rotational spring which causes discontinuity in the rotations due to bending moments. In the present work, columns with various end conditions such as pinned ends, fixed ends, fixed-pinned ends and fixed-free ends are studied. The analysis depends on the assumed deflection function, which satisfies exactly the end conditions. The buckling loads for each case of end conditions for a column with arbitrary number of cracks along the column length are given in simple and practical equations which also gave accurate results.

A comparison between the results of the present analysis and that of some previous studies shows the accuracy and the simplicity of the developed method to find the critical load in such cases.

\section{Keywords:}

Buckling loads, Stability, Columns, Spring and Crack.

* Professor, Faculty of Engineering, Minia University

** Lecturer, Faculty of Engineering, Kafrelsheikh University 


\section{Introduction:}

Columns are important structural elements such that the stability of columns under different cases of loading is investigated in many researches and theses to obtain the critical buckling loads and the critical stresses. The critical compressive load for the classical Euler column under various end conditions was obtained and discussed by Timoshenko and Gere (1) and Handbook of Structural Stability (2). Columns of uniform cross section may be containing a single crack or multiple cracks at any arbitrary sections. The cracks may develop from impact, applied cyclic loads, mechanical vibrations, aerodynamic loads etc. The stability of compressed column may be decreased due to the effect of weakness occurred in the cracked sections. The critical buckling loads of cracked columns are an important to study to investigate the effect of depths, locations and number of cracks. Cracked section is modelled by a massless rotational spring. Buckling of weakened column at an interior location (which modeled using a massless rotational spring) was analyzed by C. Y. Wang, C. M. Wang and Tun Myint Aung [3]. Buckling of slender prismatic columns with single edge crack under concentric vertical loads was studied by M. Arif Gurel and Murat Kisa [4] using the transfer matrix method. Also, by M. Arif Gurel [5] the buckling of slender prismatic circular columns weakened by multiple edge cracks was studied.

In this thesis, by using the minimum potential energy technique, the stability of slender columns weakened by multiple edge cracks subjected to concentrated loads applied at the ends is investigated. Columns with various end conditions such as pinned ends, fixed ends, fixed-pinned ends and fixed-free ends are studied. Numerical examples are given to provide information about the effects of the boundary conditions, the location and depth of the cracks on the buckling loads of columns. Also, a comparison of the results with the previous theoretical studies is given in this thesis.

\section{Theoretical Investigation:}

\subsection{Assumptions:}

Consider a slender prismatic column with a rectangular cross section subjected to a concentrated axial load $P$ and having multiple non-propagating edge cracks of various depths $a_{i}=\zeta h$ at variable positions $x_{c i}=\beta_{\mathrm{i}} L(i=1,2, \ldots, n)$ as shown in Fig. (1). The assumptions of the present analysis are:

- The material, of which the column is made, is linearly elastic.

- The column is of constant cross section with a moment of inertia $I$.

- No local buckling at any cross-section along the column length is allowed.

- The column is perfectly straight (i.e. has no initial imperfections). 


\subsection{Deflection Function}

One term only of the Fourier series for the deflection function, which satisfies the geometric conditions, gives an exact solution for intact columns and gives a good approximation for the columns weakened by cracked sections.

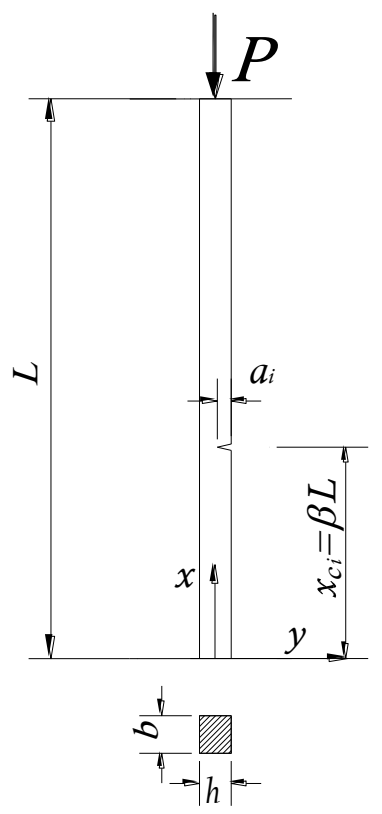

Figure (1): A slender column with multiple edge cracks

\subsection{Mathematical model}

The cracked section is modelled by a mass less rotational spring with flexibility $\mathrm{C}$. This model enables discontinuity in rotations. The restraining moment of the rotational spring is to be proportional to the difference of the junction slopes of the two segments where

$$
\Delta \theta=C M
$$

The quantity $\mathrm{C}$ is a function of the crack depth and height of the rectangular cross section of the column, and can be written as suggested in Ref. [6] as below:

$$
C=5.346 h f(\zeta)
$$

Where $h$ is the height of the cross section of the column and $\zeta=a / h$, where $a$ is the depth of the crack. The local flexibility function $f(\zeta)$ with the normalized crack depth $a / h$ for a prismatic column of rectangular cross section with a single non-propagating edge crack, is given in Ref. [6] as below:

$$
\begin{aligned}
& f(\zeta)=1.8624 \zeta^{2}-3.95 \zeta^{3}+16.375 \zeta^{4}-37.226 \zeta^{5}+76.81 \zeta^{6}-126.9 \zeta^{7} \\
& +172 \zeta^{8}-143.97 \zeta^{9}+66.56 \zeta^{10}
\end{aligned}
$$




\subsection{Method of Analysis and Theoretical Equations}

It can be seen from Fig.(1) that the column is divided into two segments, segment 1 $\left(0 \leq x \leq x_{\mathrm{c}}\right)$ and segment $2\left(x_{\mathrm{c}} \leq x \leq L\right)$, by the rotational spring. There is continuity of displacements, bending moments and shear forces at the boundary of the first and second segments, but there is a discontinuity between slopes at this point caused by the bending moment and rotation of the spring as follows

$$
\begin{aligned}
& \theta_{2}\left(x_{1}\right)-\theta_{1}\left(x_{1}\right)=y_{2}^{\prime}\left(x_{1}\right)-y_{1}^{\prime}\left(x_{1}\right) \\
& \Delta \theta\left(x_{1}\right)=C y_{1}^{\prime \prime}\left(x_{1}\right)=-C \frac{M\left(x_{1}\right)}{E I}
\end{aligned}
$$

The strain energy due to the effect of the rotational spring is

$$
\begin{aligned}
& \Delta U=\frac{1}{2} M\left(x_{1}\right) \Delta \theta\left(x_{1}\right)=-\frac{C}{2} \frac{M^{2}\left(x_{1}\right)}{E I} \\
& \Delta U=-\frac{C}{2} E I y^{\prime \prime 2}\left(x_{1}\right)
\end{aligned}
$$

\subsubsection{Column with Pinned Ends ( P-P Column)}

Assuming the following deflection function, which satisfies the end conditions

$$
y=A \sin \frac{\pi}{l} x
$$

Substituting in the energy equations, the strain energy is as follows

$$
\begin{gathered}
U=\int_{0}^{l} \frac{M^{2}}{2 E I} d x+\Delta U \\
M=P . y=P A \sin \frac{\pi}{l} x \\
U=\frac{P^{2} A^{2} l}{4 E I}+\frac{C_{1} P^{2} A^{2}}{2 E I} \sin ^{2} \frac{\pi}{l} x_{1}
\end{gathered}
$$

For $N$ cracked sections in the column, the strain energy will be

$$
U=\frac{P^{2} A^{2} l}{4 E I}\left[1+\sum_{i=1}^{N} \frac{2 C_{i}}{l} \sin ^{2} \frac{\pi}{l} x_{i}\right]
$$


The work done $T$ due to the axial load $P$ is given by

$$
\begin{aligned}
& T=\frac{P}{2} \int_{0}^{l} y^{\prime 2} d x \\
& T=\frac{P A^{2} \pi^{2}}{4 l}
\end{aligned}
$$

The critical buckling load can be obtained by the equation

$$
U=T
$$

Which represents the condition when the equilibrium configuration changes from stable to unstable mode

From equations (11) and (13), one can obtain

$$
P_{c r}=\mu \frac{\pi^{2} E I}{l^{2}}
$$

Where,

$$
\mu=1 /\left(1+\sum_{i=1}^{N} \frac{2 C_{i}}{l} \sin ^{2} \frac{\pi}{l} x_{i}\right)
$$

\subsubsection{Column with Clamped Ends (C-C Column)}

Assuming the following deflection function, which satisfies the end conditions

$$
y=A \sin ^{2} \frac{\pi}{l} x
$$

Substituting in the energy equations, the strain energy is as follows

$$
\begin{gathered}
M=P A / 2-P \cdot y=P A / 2 \cos \frac{2 \pi}{l} x \\
U=\frac{P^{2} A^{2} l}{16 E I}-\frac{C_{1} P^{2} A^{2}}{8 E I} \cos ^{2} \frac{2 \pi}{l} x_{1}
\end{gathered}
$$

For $N$ cracked sections in the column, the strain energy will be

$$
U=\frac{P^{2} A^{2} l}{16 E I}\left[1+\sum_{i=1}^{N} \frac{2 C_{i}}{l} \cos ^{2} \frac{2 \pi}{l} x_{i}\right]
$$

The work done $T$ due to the axial load $P$ is given by

$$
\begin{gathered}
T=\frac{P}{2} \int_{0}^{l} y^{\prime 2} d x \\
T=\frac{P A^{2} \pi^{2}}{4 l}
\end{gathered}
$$


The critical buckling load can be obtained by the equation

$$
U=T
$$

From equations (19) and (21), one can obtain

$$
P_{c r}=\mu \frac{4 \pi^{2} E I}{l^{2}}
$$

Where,

$$
\mu=1 /\left(1+\sum_{i=1}^{N} \frac{2 C_{i}}{l} \sin ^{2} \frac{\pi}{l} x_{i}\right)
$$

\subsubsection{Column with One End Clamped and the Other End Free (C-F Column)}

Assuming the following deflection function, which satisfies the end conditions

$$
y=A\left(1-\cos \frac{\pi}{2 l} x\right)
$$

Substituting in the energy equations, the strain energy is as follows

$$
\begin{aligned}
& M=P(A-y)=P A \cos \frac{\pi}{2 l} x \\
& U=\frac{P^{2} A^{2} l}{4 E I}-\frac{C_{1} P^{2} A^{2}}{2 E I} \cos ^{2} \frac{\pi}{2 l} x_{1}
\end{aligned}
$$

For $N$ cracked sections in the column, the strain energy will be

$$
U=\frac{P^{2} A^{2} l}{4 E I}\left[1+\sum_{i=1}^{N} \frac{2 C_{i}}{l} \cos ^{2} \frac{\pi}{2 l} x_{c i}\right]
$$

The work done $T$ due to the axial load $\mathrm{P}$ is given by

$$
\begin{gathered}
T=\frac{P}{2} \int_{0}^{l} y^{\prime 2} d x \\
T=\frac{P A^{2} \pi^{2}}{4 l}
\end{gathered}
$$

The critical buckling load can be obtained by the equation

$$
U=T
$$

From equations (27) and (29), one can obtain

$$
P_{c r}=\mu \frac{\pi^{2} E I}{4 l^{2}}
$$

Where, $\quad \mu=1 /\left(1+\sum_{i=1}^{N} \frac{2 C_{i}}{l} \cos ^{2} \frac{\pi}{2 l} x_{c i}\right)$ 


\subsubsection{Column with One End Clamped and the Other End Pinned (C-P Column)}

Assuming the following deflection function, which satisfies the end conditions

$$
y=A(\sin k x-k x-k l \cos k x+k l) \quad k l=4.493
$$

Substituting in the energy equations, the strain energy is as follows

$$
\begin{gathered}
y^{\prime \prime}=A k^{2}(k l \cos k x-\sin k x) \\
U=4.493 E I A^{2} k^{4}\left(l+C_{1} E I A^{2} k^{4}\left(k^{2} l^{2} \cos ^{2} k x_{c}+\sin ^{2} k x_{c}-k l \sin 2 k x_{c}\right)\right.
\end{gathered}
$$

For $N$ cracked sections in the column, the strain energy will be

$$
U=4.493 E I A^{2} k^{4}\left(l+\sum_{i=1}^{N} \frac{C_{i}}{2} E I A^{2} k^{4}\left(k^{2} l^{2} \cos ^{2} k x_{c i}+\sin ^{2} k x_{c i}-k l \sin 2 k x_{c i}\right)\right.
$$

The work done $T$ due to the axial load $P$ is given by

$$
\begin{aligned}
& T=\frac{P}{2} \int_{0}^{l} y^{\prime 2} d x \\
& T=4.493 P A^{2} k^{2} l
\end{aligned}
$$

The critical buckling load can be obtained by the equation

$$
U=T
$$

From equations (35) and (37), one can obtain

$$
P_{c r}=\mu \frac{(2.05) \pi^{2} E I}{l^{2}}
$$

Where, $\quad \mu=1-\sum_{i=1}^{N} \frac{C_{i}}{2 k l^{2}}\left(k^{2} l^{2} \cos ^{2} k x_{c i}+\sin ^{2} k x_{c i}-k l \sin 2 k x_{c i}\right)$

\section{Results and Discussion}

In order to see more clearly the effects of crack depth $(a=\zeta h)$ and the location of the crack $\left(x_{c}=\beta L\right)$, consider four compression columns having a single edge crack and having pinned-pinned, fixed-free, fixed-pinned and fixed-fixed support conditions. The columns have the same cross section dimensions of $h=0.1 \mathrm{~m}, b=0.1 \mathrm{~m}$ but different lengths of $1.30,0.65,1.85$ and $2.60 \mathrm{~m}$ respectively. With these properties all columns buckle in the elastic range. The first critical buckling load to the Euler buckling load ratio versus crack location parameter curves, corresponding to the $0.20,0.30$ and 0.40 values of the crack depth parameter, are plotted in Figs. (2a) to (2d). 


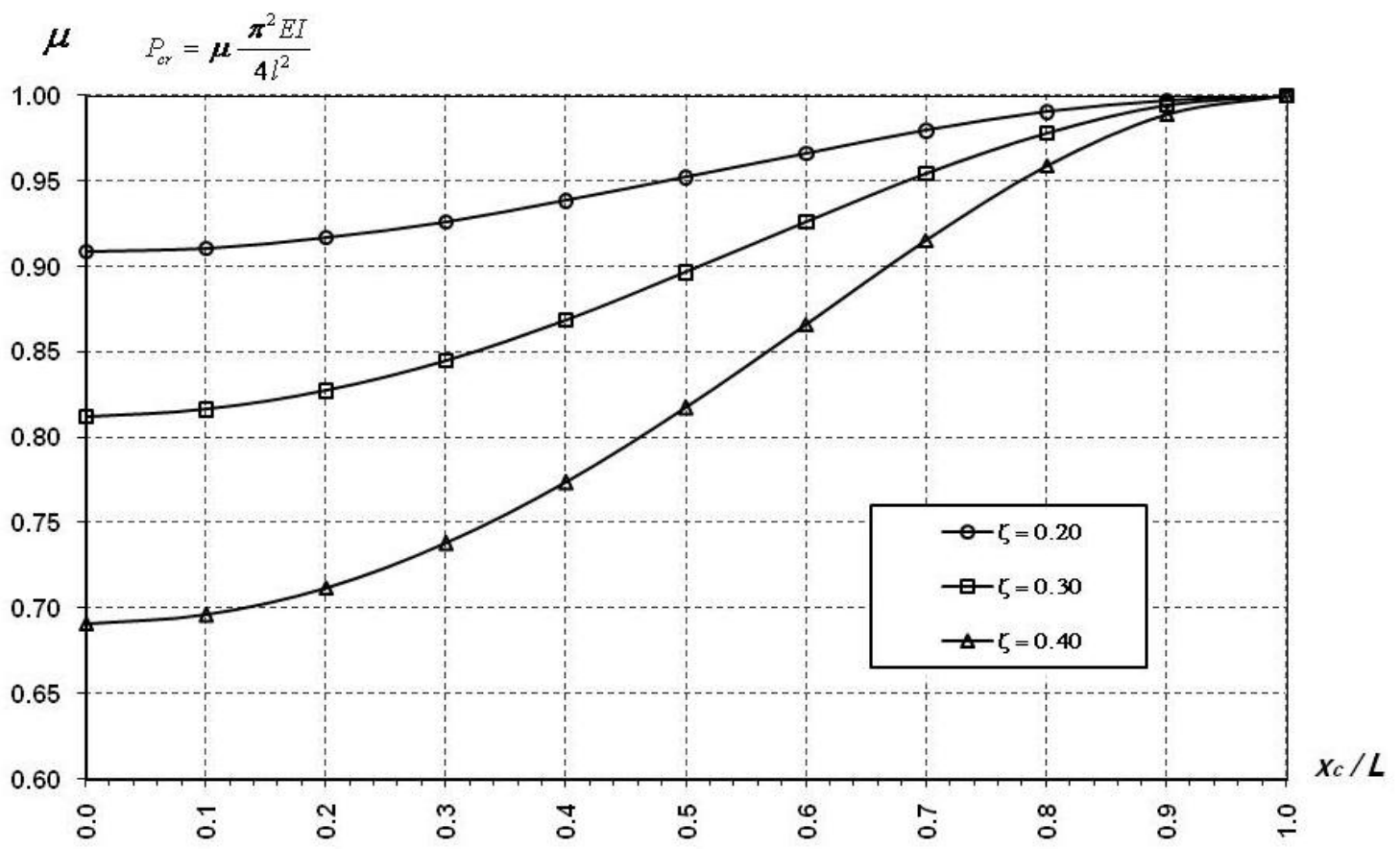

Figure (2a): Critical buckling load coefficient $(\mu) \quad$ [Clamped-free ends]

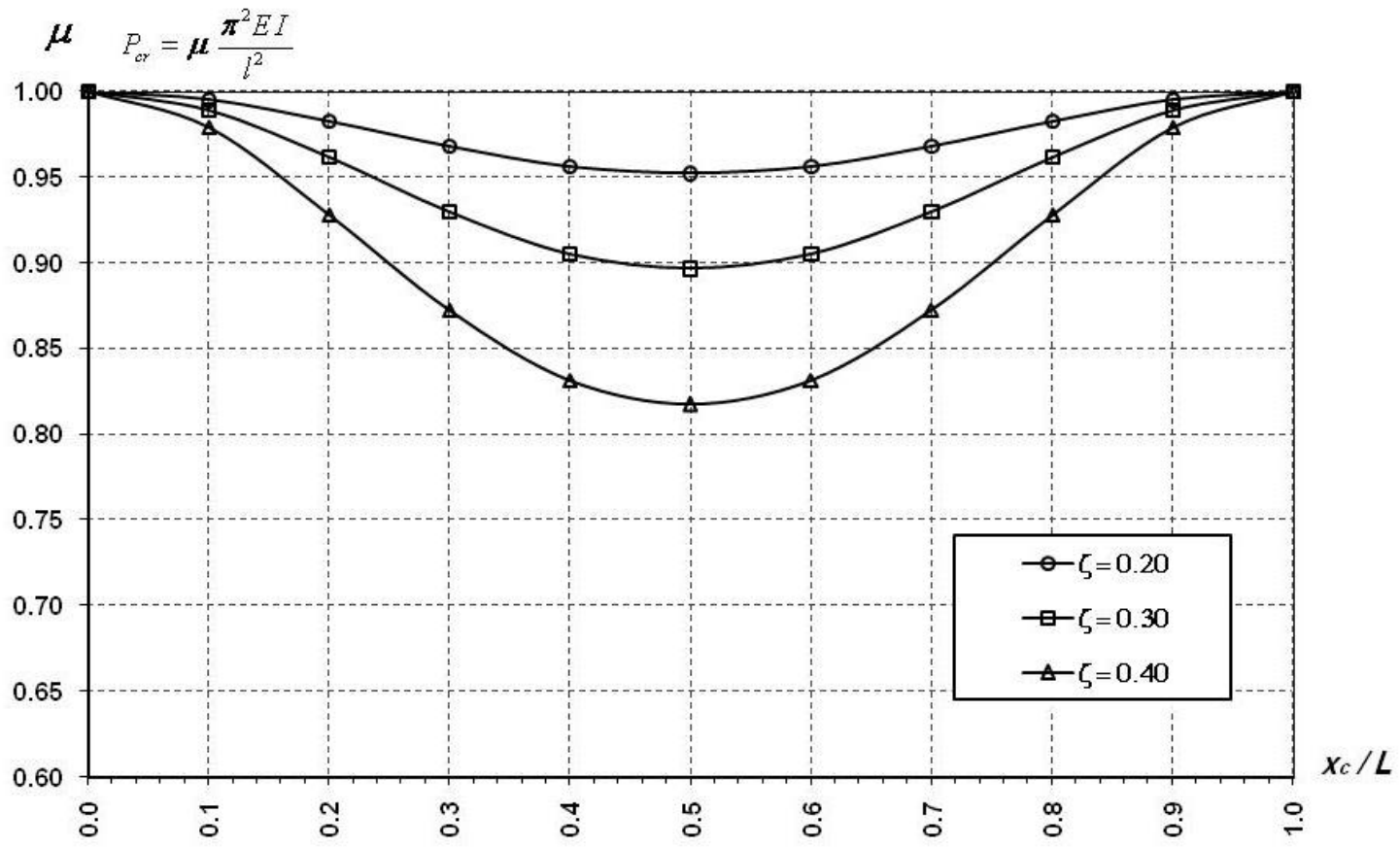

Figure (2b): Critical buckling load coefficient ( $\mu$ ) [Pinned-pinned ends] 


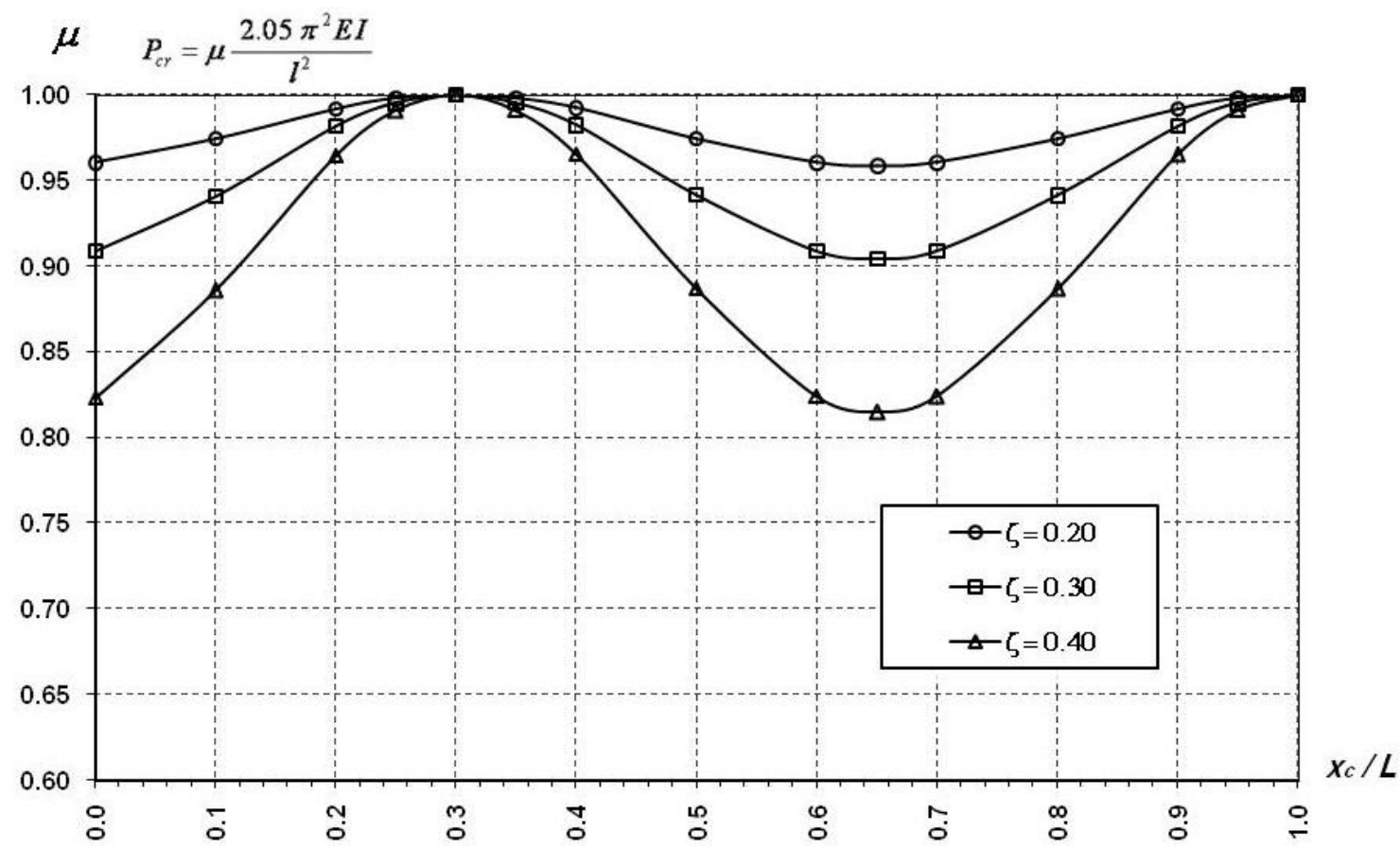

Figure (2c): Critical buckling load coefficient $(\mu) \quad$ [Clamped-pinned ends]

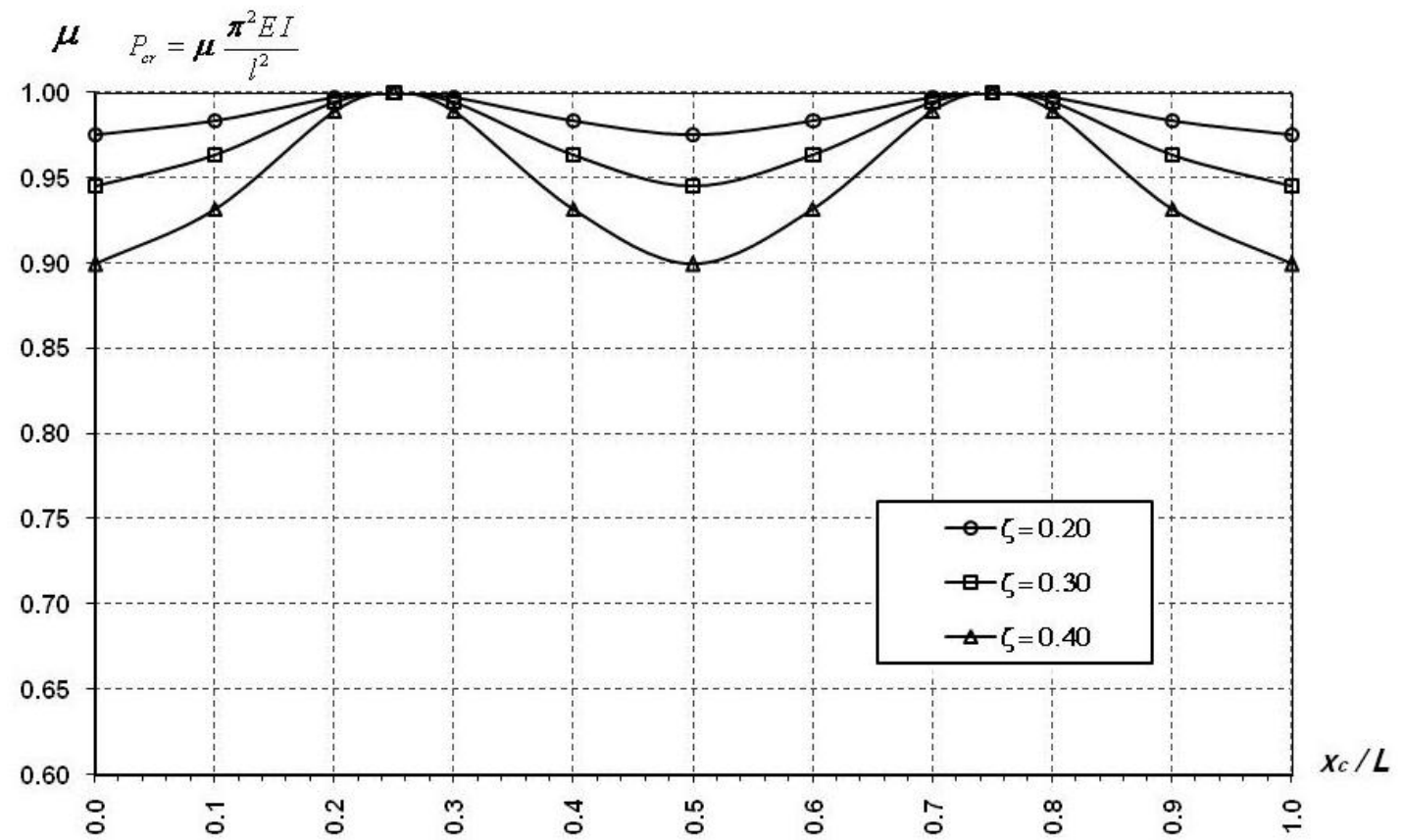

Figure (2d): Critical buckling load coefficient $(\mu) \quad$ [Clamped-clamped ends] 


\section{Comparison of the Results:}

To check the accuracy of the present analysis, comparison of the results obtained from the present work with the theoretical results of some previous studies is carried out. Two numerical examples of 2 specimen columns shown in Fig.(3) which solved by the transfer matrix method [4] to obtain the critical buckling load will be resolved using the present work.

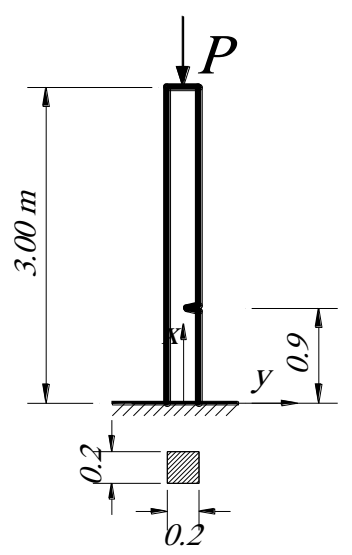

(a) Clamped- Free column

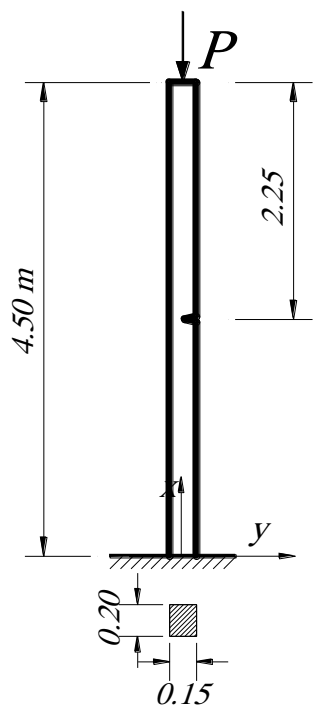

(b) Pinned-pinned column

Figure (3): Columns considered as numerical examples

Example 1: a clamped- free ended column shown in Fig.(3a) with the following data is considered

$h=b=20 \mathrm{~cm}, L=3 \mathrm{~m}, a=0.3 \mathrm{~h}=6 \mathrm{~cm}$ and $\mathrm{xc}=0.3 L=0.90 \mathrm{~m}$ from the clamped end $\zeta=a / h=0.30 \gg>\mathrm{C}=0.149935$, then the buckling load obtained as

$$
P_{c r}=0.926479 \frac{\pi^{2} E I}{4 L^{2}}
$$

If the depth of the crack increased to $a=0.45 h=9.0 \mathrm{~cm}$, then the buckling load obtained as

$$
P_{c r}=0.827503 \frac{\pi^{2} E I}{4 L^{2}}
$$

If the location of the crack is shifted to $x_{c}=0.05 \mathrm{~L}$, then

$$
P_{c r}=0.909635 \frac{\pi^{2} E I}{4 L^{2}}
$$


Example 2 : a pinned - pinned ended column shown in Fig.(3b) with the following data is considered

$h=15 \mathrm{~cm}, b=20 \mathrm{~cm}, L=4.5 \mathrm{~m}, a=0.25 h=3.75 \mathrm{~cm}$ and $x_{c}=0.5 L=2.25 \mathrm{~m}$

$\zeta=a / h=0.25 \gg>\mathrm{C}=0.076532$, then the buckling load obtained as

$$
P_{c r}=0.967105 \frac{\pi^{2} E I}{L^{2}}
$$

If the depth of the crack increased to $a=0.50 h=7.5 \mathrm{~cm}$, then the buckling load obtained as

$$
P_{c r}=0.84964 \frac{\pi^{2} E I}{L^{2}}
$$

If the location of the crack is shifted to $x_{c}=0.15 \mathrm{~L}$, then

$$
P_{c r}=0.993038 \frac{\pi^{2} E I}{L^{2}}
$$

Table (1) shows a comparison between the resulting values of the factor $\mu$ of the two previous examples for various values of $\zeta$ and $\beta$ with the resulting values obtained by the transfer matrix method [4].This comparison clears the satisfactory accuracy of the

\begin{tabular}{|c|c|c|c|c|}
\hline \multicolumn{2}{|c|}{ Example } & $\begin{array}{c}\text { Ref. } \\
{[4]}\end{array}$ & $\begin{array}{c}\text { Present } \\
\text { Work }\end{array}$ & $\begin{array}{l}(\%) \\
\text { Diff. }\end{array}$ \\
\hline \multirow{3}{*}{$\begin{array}{c}\text { Example (1) } \\
\text { Clamped - Free } \\
\begin{array}{c}L=3.00 \mathrm{~m} \\
h=20 \mathrm{~cm} \\
b=20 \mathrm{~cm}\end{array}\end{array}$} & $\begin{array}{l}\zeta=0.30 \\
\beta=0.30\end{array}$ & 0.926029 & 0.926479 & $0.05 \%$ \\
\hline & $\begin{array}{l}\zeta=0.45 \\
\beta=0.30\end{array}$ & 0.825261 & 0.827503 & $0.27 \%$ \\
\hline & $\begin{array}{l}\zeta=0.30 \\
\beta=0.05\end{array}$ & 0.908170 & 0.909635 & $0.16 \%$ \\
\hline \multirow{3}{*}{$\begin{array}{c}\text { Example (2) } \\
\text { Pinned - Pinned } \\
\begin{array}{c}L=4.50 \mathrm{~m} \\
h=15 \mathrm{~cm} \\
b=20 \mathrm{~cm}\end{array}\end{array}$} & $\begin{array}{l}\zeta=0.25 \\
\beta=0.50\end{array}$ & 0.966840 & 0.967105 & $0.03 \%$ \\
\hline & $\begin{array}{l}\zeta=0.50 \\
\beta=0.50\end{array}$ & 0.844800 & 0.849640 & $0.57 \%$ \\
\hline & $\begin{array}{l}\zeta=0.25 \\
\beta=0.85\end{array}$ & 0.992920 & 0.993038 & $0.01 \%$ \\
\hline
\end{tabular}
present work.

Table (1): Comparison of the buckling load coefficient ( $\mu$ ) with Ref.[4] 
It can be noticed that although the present equations are simple, it gave accurate results when compared with the solution by the transfer matrix method, which solved by trial and error in each particular case. Then, the present equations can be rather used by the designer engineers.

\section{Conclusions:}

In this paper, the theoretical analysis using the concept of minimum potential energy is developed to determine the critical buckling loads for slender columns weakened by multiple edge cracks subjected to concentrated loads applied at the ends and having different end conditions. A relatively simple but accurate method is presented for the prediction of the buckling load, hence the buckling stresses, for columns under study.

From the previous results the following conclusions may be drawn

1. The critical buckling load for a column with internal weakness due to one crack or multiple cracks at arbitrary positions along the column length can be accurately determined through explicit forms by means of energy solution tacking into account one term of the Fourier series in the deflection function. The proposed method of analysis is a simple and sufficient method for applications with various end conditions.

2. The critical buckling load decreases due to the presence of the cracked sections. The percentage of the reduction of the buckling load depends on the depth and the location of the crack.

3. The load carrying capacity of an axially loaded column decreases as the crack depth increases. On the other hand, the effect of the crack location on the buckling load influences by the end conditions of the compression member. Generally, when the cracked sections lie at the points of smaller bending moments the minimum effect on the buckling load will occurs and there is no effect of the cracked sections on the buckling load if it lie just at the zero moments locations (inflection points). On the opposite, the reduction of the critical buckling load will increase with the movement of the cracked section towards the maximum bending moment points.

Finally, the present analysis provides sets of simple equations which can be of great help for design purposes in cases of columns under study. The comparison of the results with previous studies confirms the accuracy of these equations. 


\section{References:}

[1] Timoshenko, S. P., and Gere, J. M. Theory of Elastic Stability, McGraw-Hill Book Company, London, (1983).

[2] Column Research Committee of Japan Handbook of Structural Stability, Book Corona, Tokyo, (1971).

[3] C. M. Wang, C. Y. Wang and Tun Myint Aung, Buckling of a Weakened Column, Journal of Engineering Mechanics, ASCE, Vol. 130, November, (2004).

[4] M. Arif Gurel and Murat Kisa, Buckling of Slender Prismatic Columns with a Single Edge Crack under Concentric Vertical Loads, Turkish J. Engng. Env. Sci., 29, 185-193 (2005).

[5] M. Arif Gurel, Buckling of Slender Prismatic Circular Columns Weakened by Multiple Edge Cracks, Acta Mech 188, 1-19 (2007).

[6] Shifrin, E.I. and Ruotolo, R., Natural Frequencies of a Beam with an Arbitrary Number of Cracks, Journal of Sound and Vibration, 222(3), 409-423, 1999.

[7] Fan, S. C., Zheng, D. Y., Stability of a cracked Timoshenko beam-column by modified Fourier, series. J. Sound Vibr. 264, 475-484 (2003).

[8] K. Yazdchi, A. R. Gowhari Anaraki, Carrying capacity of edge-cracked columns under concentric vertical loads, Acta Mech 198, 1-19 (2008).

\section{Nomenclatures:}

$A_{i}$ constant

$E$ Young's modulus

$I_{\mathrm{i}}$ moment of inertia in the ith interval

$P_{\text {cr }}$ critical buckling load

$P_{E} \quad$ Euler buckling load

$U$ strain energy

$T$ work done

$M$ bending moment

$(\mathrm{x}, \mathrm{y})$ cartesian coordinante

$y$ deflection curve

$L$ length of the column

$\mu$ buckling load coefficient 